\title{
Tools for Image Analysis and First Wall Protection at W7-X
}

Fabio Pisano, ${ }^{\mathrm{a} *}$ Barbara Cannas, ${ }^{\text {a }}$ Alessandra Fanni, ${ }^{\text {a Giuliana Sias, }}{ }^{\text {a }}$ Marcin

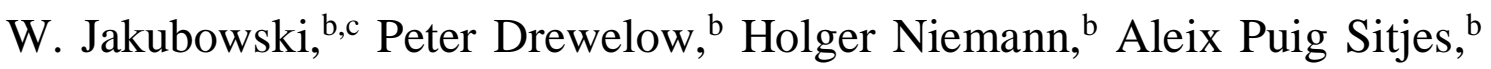

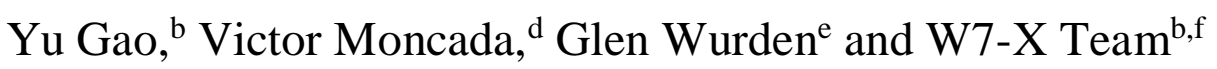

${ }^{a}$ University of Cagliari, Department of Electrical and Electronic Engineering, Cagliari, Italy

${ }^{b}$ Max-Planck-Institut für Plasmaphysik, Teilinstitut Greifswald, Greifswald, Germany

${ }^{c}$ University of Szczecin, Institute of Physics, Szczecin 70-451, Poland

${ }^{d}$ CEA, IRFM, Saint Paul lez Durance, France

${ }^{e}$ Los Alamos National Laboratory, Los Alamos, New Mexico, USA

${ }^{f}$ See the authors list in R. C. Wolf et al., Nucl. Fusion 57, 102020 (2017)

This submission is part of the 2019 IAEA TM on Fusion Data Processing, Validation and Analysis.

Correspondence details for corresponding author:

Dr. Fabio Pisano

Department of Electrical and Electronic Engineering

University of Cagliari

Piazza D'Armi, 09123 Cagliari, Italy

Phone: +39070 6755898

FAX: +39070 6755900

*Email: fabio.pisano@diee.unica.it 


\title{
Tools for Image Analysis and First Wall Protection at W7-X
}

Fabio Pisano, ${ }^{\text {a* }}$ Barbara Cannas, ${ }^{\mathrm{a}}$ Alessandra Fanni, ${ }^{\mathrm{a}}$ Giuliana Sias, ${ }^{\mathrm{a}}$ Marcin W. Jakubowski, ${ }^{\mathrm{b}, \mathrm{c}}$ Peter Drewelow, ${ }^{\mathrm{b}}$ Holger Niemann, ${ }^{\mathrm{b}}$ Aleix Puig Sitjes, ${ }^{\mathrm{b}}$ Yu Gao, ${ }^{\mathrm{b}}$ Victor Moncada, ${ }^{\mathrm{d}}$ Glen Wurden ${ }^{\mathrm{e}}$ and W7-X Team ${ }^{\mathrm{b}, \mathrm{f}}$

${ }^{a}$ University of Cagliari, Department of Electrical and Electronic Engineering, Cagliari, Italy

${ }^{b}$ Max-Planck-Institut für Plasmaphysik, Teilinstitut Greifswald, Greifswald, Germany

${ }^{c}$ University of Szczecin, Institute of Physics, Szczecin 70-451, Poland

${ }^{d}$ CEA, IRFM, Saint Paul lez Durance, France

${ }^{e}$ Los Alamos National Laboratory, Los Alamos, New Mexico, USA

${ }^{f}$ See the authors list in R. C. Wolf et al., Nucl. Fusion 57, 102020 (2017)

\begin{abstract}
Wendelstein 7-X (W7-X) is the most advanced operating stellarator in fusion research. The monitoring and control of the heat loads on the plasma facing components (PFCs) will be critical during the next operation phase, in which the ten water-cooled divertors will have to withstand heat loads of the order of $10 \mathrm{MW} / \mathrm{m}^{2}$. This paper gives an overview of the image analysis tools for the camera spatial calibration and scene modelling, which are an essential part of the real-time PFCs protection system currently under development at W7-X. Some applications of these tools are also provided, specifically for the strike-line characterization.
\end{abstract}

Keywords: Image Processing, Thermography, Plasma Facing Components

\section{INTRODUCTION}

From the next W7-X operational campaign, named OP2 [1], which is expected to start at the end of 2021, ten High Heat-Flux (HHF) water-cooled divertor units will be used for power and particle exhaust, with operation times up to 30 minutes. These units are designed to withstand $10 \mathrm{MW} / \mathrm{m}^{2}$ in steady-state. By then, a real-time PFCs monitoring system will be required, able to analyse the thermographic data, detect 
dangerous thermal events [2] and send alarms to the control system in order to prevent damage to the device. In this context, a fast spatially precise image analysis of the infrared camera data is essential.

During the last operational campaign, named OP1.2, concluded in October 2018, the imaging system under development at W7-X has been tested on the analysis of IR data, where nine immersion tubes and one endoscope were used to look at the ten inertially-cooled test divertors [3][4]. In this paper, an overview of the developments on the camera spatial calibration and mapping tools is proposed.

The paper is organized as follows. Section II gives an overview of the imaging system under development at W7-X, with a description of the thermographic diagnostics which will be used during the next operational campaign. Section III describes the camera spatial calibration process, used to create IR camera projection models. Section IV describes two types of mapping tools used at W7-X. Section V gives an overview of the possible applications of these tools with particular attention to the strike-line characterization. Finally, in Sec. VI, the conclusions and the future work outlook are given.

\section{IMAGING SYSTEM}

During OP2, 10 endoscopes with infrared and video cameras will be used to monitor the ten divertors [3]. A real-time system is under development at W7-X for the acquisition and analysis of the thermographic data coming from the endoscopes. The data will be acquired and analysed by means of computer vision techniques implemented on a GPU, which should be able to send an alarm to the interlock system in case of dangerous events and stop the operation. The acquired data will be sent to the control room for visualization and control purposes, and the detected thermal events will be stored in a thermal events database [4]. 
In the framework of data acquisition and analysis, the temperature calibration [2] and the calculation of the heat load distribution in the target surfaces [5] require knowledge about the observed materials and their emissivity, and explicit information about the position of the observed surface and the line of sight with respect to the camera eye. All this information is stored in the so-called scene models (see section IV.A), which contain a pixel-based segmentation of the field of view, giving information about the material and spatial properties of the target surfaces. For the creation of the scene models, it is necessary to build a projection model of the camera through a spatial calibration process (see section III).

Figure 1 shows an overview of the full processing pipeline, starting from the raw images coming from the IR cameras up to the heat flux evaluation on the target surfaces. The main inputs of the process are the raw IR images and the CAD model of the PFCs. By means of these inputs, the IR cameras are calibrated spatially, and a camera model (see section III.a) is generated. The camera model is then used to create the scene model. From the information taken from the scene model, the IR camera image, where a temperature is assigned to each pixel, is created. This image can be projected by means of the scene model on the CAD view, obtaining a surface temperature for each point in the surface. By knowing the surface temperatures, the heat flux along several 1D profiles through the divertor surface is calculated by means of the THEODOR code [5] and finally the heat flux image is obtained. From the CAD model it is also possible to create a thermal map, a CAD-based 2D map which unfolds the first wall of the stellarator (see section IV.B). 


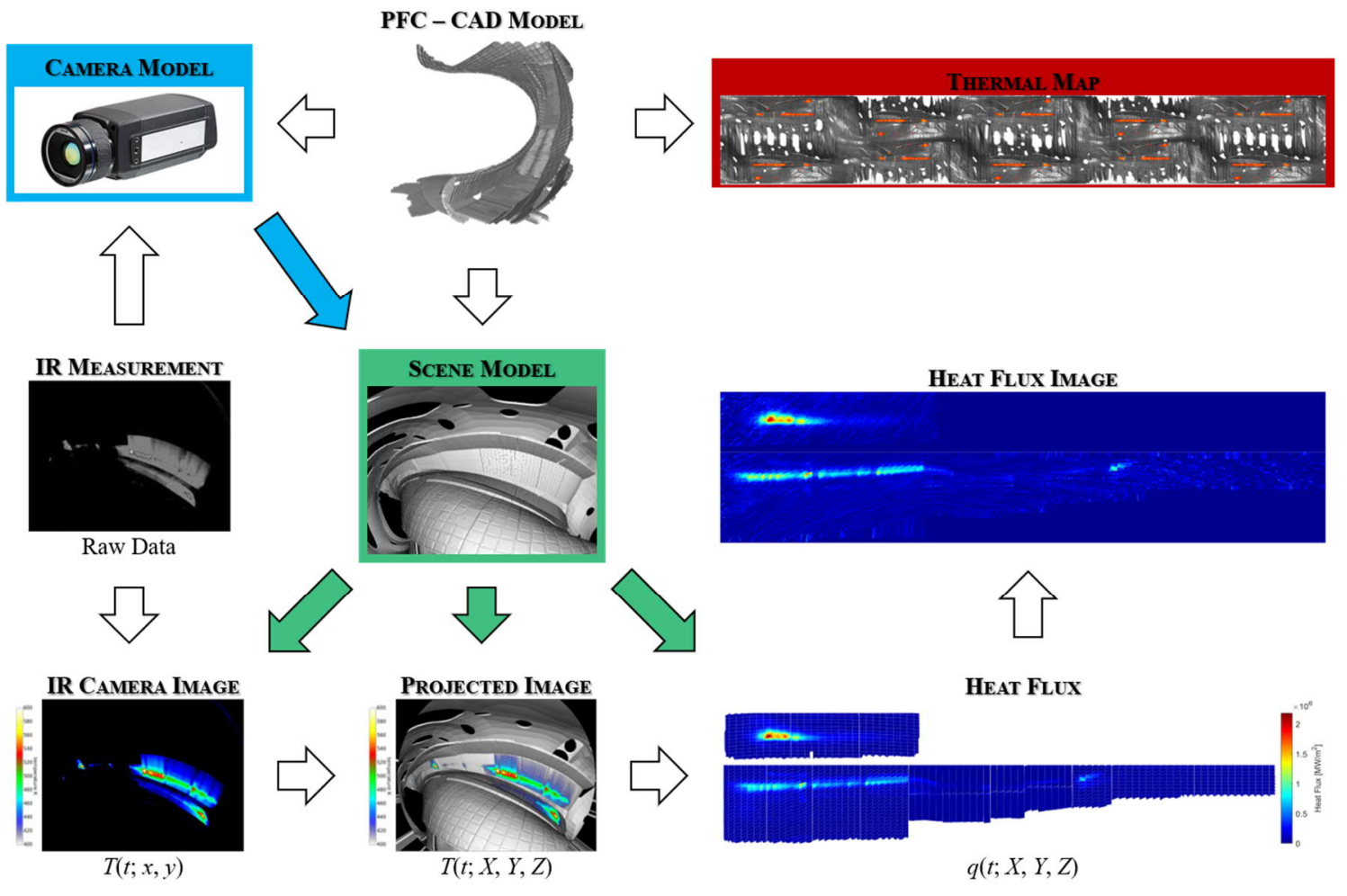

Figure 1. Overview of the imaging system, starting from raw IR measurements up to temperature and heat flux calculations.

\section{SPATIAL CALIBRATION}

Spatial calibration is an essential step in the overall process, since it helps to build a biunivocal correspondence between the position of the pixels in the image and the actual position of the 3D points in the observed surface. In fact, this step consists in finding the camera model which better fits the relationship between the known 3D coordinates of a set of control points and their projection on the image plane.

\section{III.A CAMERA MODEL}

The camera model is based on the pinhole camera model [6] [7]. Such a camera model describes the perspective of the camera as if it were an ideal pinhole camera, whose aperture is described as a point and where no lenses are used to focus light. The camera model described below is an improvement of the camera model used in [8] to model cameras during the first W7-X operational campaign. The main improvement is due to 
the new distortion function, which helps to avoid conditioning problems in case of high image distortion.

The camera model depends on several parameters: extrinsic parameters, defining the pinhole position and orientation of the equivalent pinhole in the $3 \mathrm{D}$ world; intrinsic parameters, defining the camera focal length and skew factor in pixels and the position of the principal point in the image plane; distortion parameters, defining the lens distortion effect in terms of three components, i.e. radial, tangential and prism. The thermographic diagnostic uses wide angle lenses, leading to large image distortions, whose modelling is a challenging problem.

In the camera model, the relationship between the 3D point $\boldsymbol{P}=[X, Y, Z]$ and the pixel $\boldsymbol{p}=[x, y]$ is given by

$$
\boldsymbol{p}=\frac{\boldsymbol{P}_{c}}{Z_{c}} \boldsymbol{K}_{d}\left(\boldsymbol{P}_{c}\right) \boldsymbol{K}
$$

where

$$
\boldsymbol{P}_{c}=\left(\boldsymbol{P}-\boldsymbol{P}_{0}\right) \boldsymbol{Q}
$$

In (1b), the resulting point $\boldsymbol{P}_{c}=\left[X_{c}, Y_{c}, Z_{c}\right]$ is the $3 \mathrm{D}$ point in the camera reference coordinate system, obtained through a translation of the 3D point $\boldsymbol{P}$ with respect to the pinhole position $\boldsymbol{P}_{0}$ and through a rotation by means of the camera orientation matrix $\boldsymbol{Q}$. Both $\boldsymbol{P}_{0}$ and $\boldsymbol{Q}$ represent the extrinsic parameters. The point $\boldsymbol{P}_{c}$ is then normalized in (1a) with respect to the depth coordinate $Z_{c}$, distorted by means of a distortion matrix $\boldsymbol{K}_{d}\left(\boldsymbol{P}_{c}\right)$ which models the lens distortion effect. Finally, it is transformed into a pixel point by means of the intrinsic parameters matrix $\boldsymbol{K}$. The projection scheme is shown in Figure 2. 

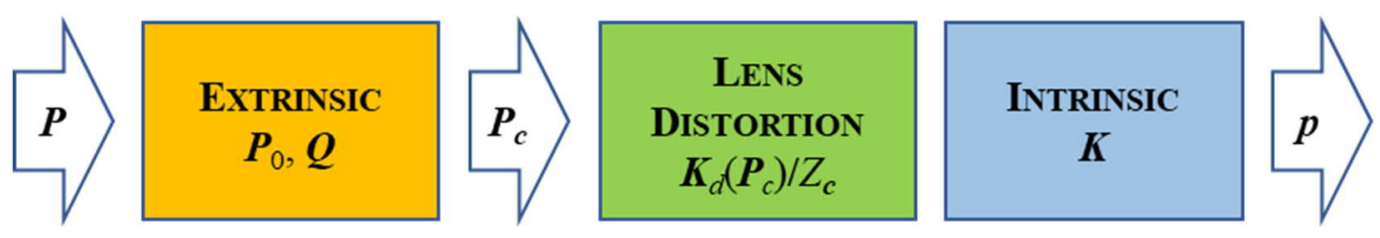

Figure 2. Projection scheme of the camera model

The distortion matrix $\boldsymbol{K}_{d}\left(\boldsymbol{P}_{c}\right)=\boldsymbol{K}_{r}\left(\boldsymbol{P}_{c}\right)+\boldsymbol{K}_{t p}\left(\boldsymbol{P}_{c}\right)$ in (1a) is a diagonal matrix given by the sum of two contributions: a radial distortion component $\boldsymbol{K}_{r}$ and a tangential-prism distortion component $\boldsymbol{K}_{t p}$.

\section{III.B RADIAL DISTORTION}

The radial distortion function has been designed in order to be: general enough to deal with both the immersion tubes and endoscope lenses used during OP1.2 experimental campaign, characterized by low distortion and very strong distortion, respectively; robust enough to overcome the conditioning problems of the state-of-the-art polynomial radial distortion models [9]. Polynomial models, due to their oscillating behaviour, which is not commonly observed in normal lenses, also overfit when interpolating and deviate completely when extrapolating, and this problem gets worse as the distortion increases.

The radial distortion matrix is given by

$$
\boldsymbol{K}_{r}\left(\boldsymbol{P}_{c}\right)=\operatorname{diag}\left(f\left(\frac{R_{c}}{Z_{c}}\right), f\left(\frac{R_{c}}{Z_{c}}\right), 1\right)
$$

where $R_{c}=\sqrt{X_{c}^{2}+Y_{c}^{2}}$ is the radial coordinate in the camera reference system and the proposed radial distortion function is

$$
\begin{gathered}
f(r)=(1-\lambda) \frac{G_{n}\left(t_{1}\right)}{t_{1}}+\lambda \frac{G_{n}\left(t_{2}\right)}{t_{2}}+ \begin{cases}0 & r \geq 0 \\
2(1-\lambda) r_{\gamma 1}+2 \lambda r_{\gamma 2} & r<0\end{cases} \\
G_{n}(t)=\int_{0}^{t} \frac{1}{1+\tau^{n / 2}} d \tau, \quad t_{1}=\frac{|r|}{r_{\gamma 1}} G_{n}(\infty), \quad t_{2}=\frac{|r|}{r_{\gamma 2}} G_{n}(\infty), \quad G_{n}(\infty)=\frac{2 \pi / n}{\sin (2 \pi / n)}
\end{gathered}
$$


The distortion model in (3) mainly depends on two knee points, $r_{\gamma 1}$ and $r_{\gamma 2}$, with $r_{\gamma 1} \leq r_{\gamma 2}$, and on the approximative slope $\lambda$ of the distorted radius $r f(r)$ between the two knee points, as shown in Figure 3. As the parameter $n \in \square$ increases, the function becomes more similar to a piecewise linear function (red dashed line in Figure 3).

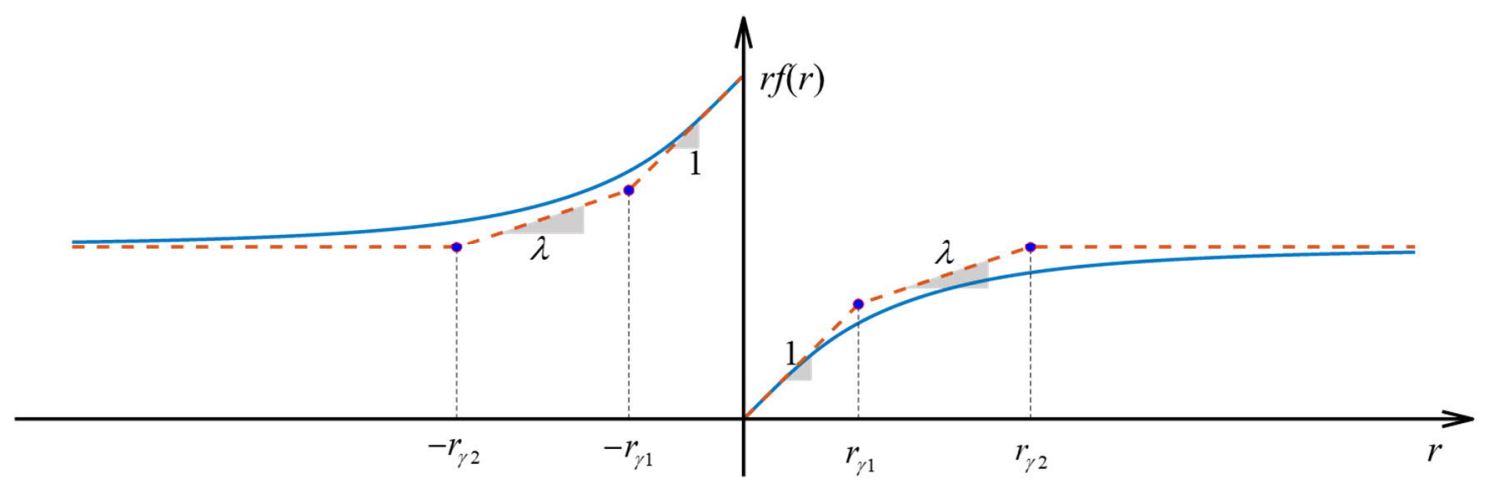

Figure 3. Function used for modelling radial distortion in fish-eye lenses.

An interesting property of this distortion function is that it includes a continuation between $r \rightarrow \infty$ and $r \rightarrow-\infty$, i.e. when $Z_{c}$ changes sign from positive to negative. In fact, it may happen that for strong fish-eye lens distortion some points closer to the camera eye have a $Z_{c}<0$, which consists in an equivalent field of view, i.e. the field of view with respect to the equivalent pinhole, slightly higher than $180^{\circ}$. Without this continuation, points beyond the standard $180^{\circ}$ field of view may remain uncovered by the model, even if they are within the camera frame. In the distortion function, the problem is treated as for spherical distortion lenses, i.e. there is an axial symmetry between what happens for $r>0$ and what happens for $r<0$.

The solution of the integral in (3) is shown below for the sake of completeness

$$
\int_{0}^{t} \frac{1}{1+\tau^{n / 2}} d \tau=\left\{\begin{array}{ll}
2\left[\sqrt{t}+\log \left(\frac{1}{1+\sqrt{t}}\right)\right] & n=1 \\
\frac{2}{n} \sum_{k=0}^{n-1} c_{k, n}^{2} \log \left(\frac{c_{k, n}}{c_{k, n}-\sqrt{t}}\right) & n>1
\end{array} \quad c_{k, n}=\exp \left(j \frac{2 k+1}{n} \pi\right)\right.
$$


where $j$ is the imaginary unit. Two particular cases, for $n=2$ and $n=4$, are shown below:

$$
\begin{aligned}
& G_{2}(t)=\int_{0}^{t} \frac{1}{1+\tau} d \tau=\log (1+t) \\
& G_{4}(t)=\int_{0}^{t} \frac{1}{1+\tau^{2}} d \tau=\operatorname{atan}(t)
\end{aligned}
$$

The first case in (5a) is compatible with the model defined as fisheye transform in [9], while the second case in (5b) is compatible with the models defined as perspective model and field-of-view model in [9]. 


\section{III.C TANGENTIAL AND PRISM DISTORTION}

The tangential-prism distortion component used in this paper is given by

$$
\boldsymbol{K}_{t p}\left(\boldsymbol{P}_{c}\right)=\operatorname{diag}\left(\frac{2 X_{c} \boldsymbol{P}_{c} \boldsymbol{k}_{t}^{T}+k_{t p x} R_{c}^{2}}{X_{c}\left(Z_{c}-2 \boldsymbol{P}_{c} \boldsymbol{k}_{t}^{T}\right)-k_{t p x} R_{c}^{2}}, \frac{2 Y_{c} \boldsymbol{P}_{c} \boldsymbol{k}_{t}^{T}+k_{t p y} R_{c}^{2}}{Y_{c}\left(Z_{c}-2 \boldsymbol{P}_{c} \boldsymbol{k}_{t}^{T}\right)-k_{t p y} R_{c}^{2}}, 0\right)
$$

where $\boldsymbol{k}_{t}=\left[k_{t x}, k_{t y}, 0\right]$ is the vector containing the tangential distortion coefficients, $k_{t p x}=k_{t x}+k_{p x}$ and $k_{t p y}=k_{t y}+k_{p y}$, with $\boldsymbol{k}_{p}=\left[k_{p x}, k_{p y}, 0\right]$ containing the prism distortion coefficients.

Note that the tangential-prism distortion matrix in (6), when the distortion parameters are very small, reduces to:

$$
\boldsymbol{K}_{t p}\left(\boldsymbol{P}_{c}\right) \cong \operatorname{diag}\left(\frac{2 X_{c} \boldsymbol{P}_{c} \boldsymbol{k}_{t}^{T}+k_{t p x} R_{c}^{2}}{X_{c} Z_{c}}, \frac{2 Y_{c} \boldsymbol{P}_{c} \boldsymbol{k}_{t}^{T}+k_{t p y} R_{c}^{2}}{Y_{c} Z_{c}}, 0\right)
$$

which is consistent with the model described in [10].

\section{III.D OPTIMIZATION}

The spatial calibration process consists normally of two main steps, one related with the optimization of intrinsic and distortion parameters of the camera, one related with the optimization of the extrinsic parameters (camera position and orientation).

For the first calibration phase, multiple views of a checkerboard-like-object (see Figure $4 a-b)$ have been collected before the experimental campaign. In order to obtain the checkerboard images, the second layer of a two-layer metal checkerboard were heated up by electrical heating stripes. The result was a clear checkerboard pattern in which the squares contour was clearly visible, without optical aliasing. A set of corner points were automatically detected from each checkerboard image, related with the reference chessboard, and used for the calibration of intrinsic and distortion parameters. In this 
procedure, the intrinsic parameters matrix is first initialized without including the distortion model by computing the homographies as proposed in [11]. After this, the radial distortion is included in the model and optimized iteratively together with the intrinsic matrix, increasing at each step the parameter $n$. The radial distortion model, which best fits in the least square sense the projection of checkerboard corners onto checkerboard images, is chosen. A final correction of the parameters is done after adding tangential and prism distortion. If these distortion parameters do not improve the fitting, then these parameters are discarded, setting them to zero.

a)

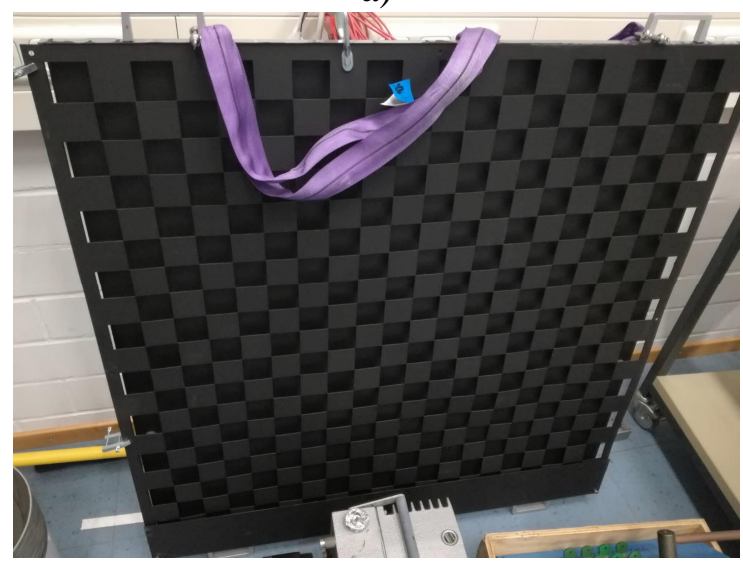

b)

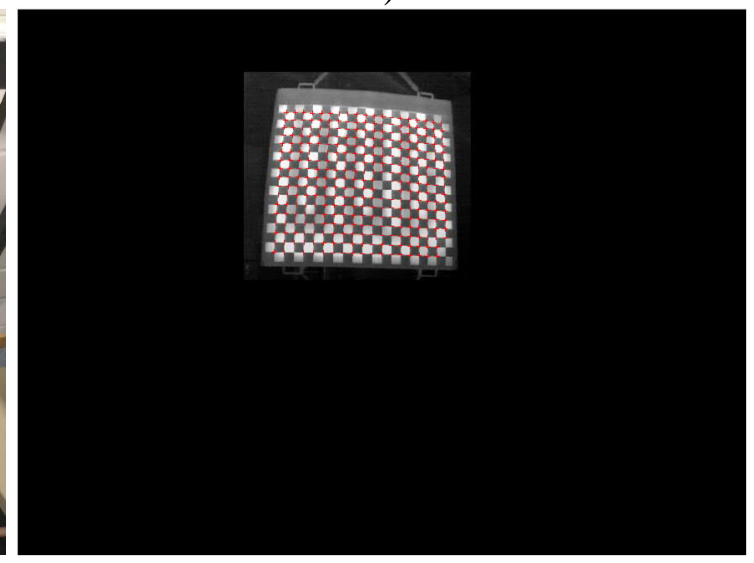

c)

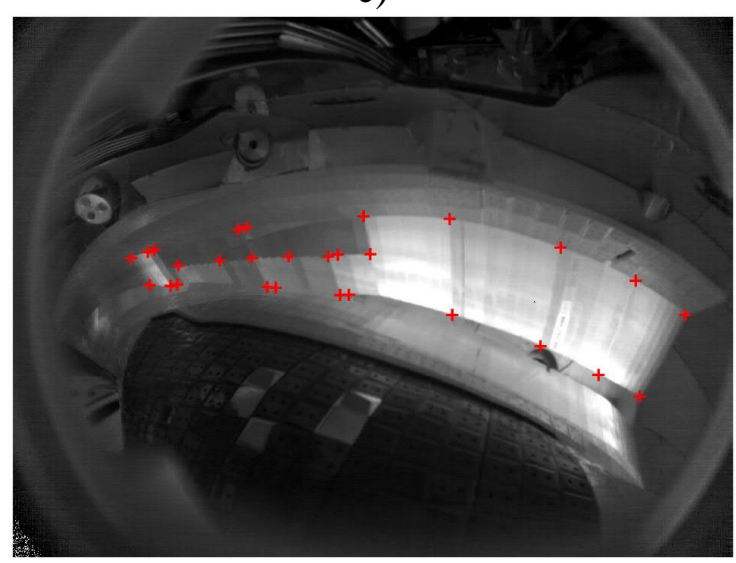

Figure 4 a) Two-layer checkerboard used to generate checkerboard images; b) Example of checkerboard image with detected corner points; c) Example of background image with selected control points. 
For the second phase, a background image (see Figure 4c) is collected every time the camera position changes. The background image is used to collect a set of control points on the observed surface. Typically, these points consist of the corners of the PFC tiles. The control points are related to the correspondent 3D point in a simplified CAD model of the PFCs and used for the calibration of extrinsic parameters. In this procedure, the extrinsic parameters are first initialized by considering the nominal position and orientation of the camera. Then, the extrinsic parameters of the full camera model, which comprises also the intrinsic and distortion parameters optimized in the previous phase, are optimized in order to best fit in the least square sense the projection of 3D CAD points onto the background image.

Regarding the error obtained at the end of the calibration procedure, it has proved to be pretty small in the divertor and baffle area, the region of interest for the heat load studies. Far away from the divertor region, in the wall tiles for example, a higher error was obtained, due also to the most prevalent distortion effect far away from the principal point.

\section{MAPPING TOOLS}

Knowing the relationship between the 3D world coordinates and the camera pixels is important in thermography to convert the photon fluxes observed by the IR sensor into surface temperatures, which depend on the emissivity of the observed materials and on the camera position and orientation. It is also important to build a relationship between the detected thermal events and the 3D model of the observed surfaces in order to determine the risk of such events. By means of the spatial calibration explained in the previous section, it is possible to carry out this task applying proper mapping tools which can be subdivided by camera-dependent and CAD-dependent. 
Scene models are an example of camera-dependent mapping tools, as they contain a pixel-based segmentation of the field of view for each camera, giving information about material and spatial properties of the target surfaces. The so-called thermal map is an example of a CAD-dependent mapping tool, since it helps to visualize all the thermal events into a single image of the PFCs bypassing the camera position dependence of the scene models.

\section{IV.A CAMERA-DEPENDENT: SCENE MODELS}

A scene model contains, for each camera, a pixel-wise information about: the observed PFC and its emissivity properties; the distance of the target material from the camera eye and the angle of the line of sight with respect to the surface normal; the 3D coordinates of the observed target [2]. It also contains a simulation of the CAD view as it would be projected onto the camera plane, as it can be seen in Figure 5a. The overlapping of the CAD view on the thermal image (Figure $5 b$ ) is used during experiments to help the visualization and interpretation of the detected thermal events with respect to the observed surfaces.

a)

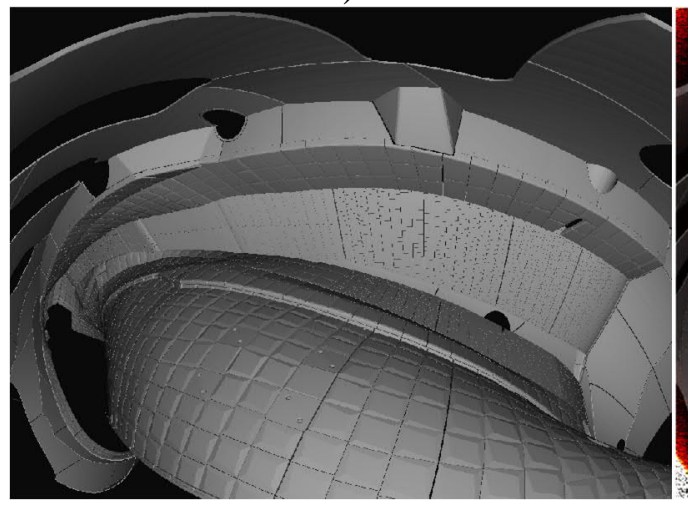

b)

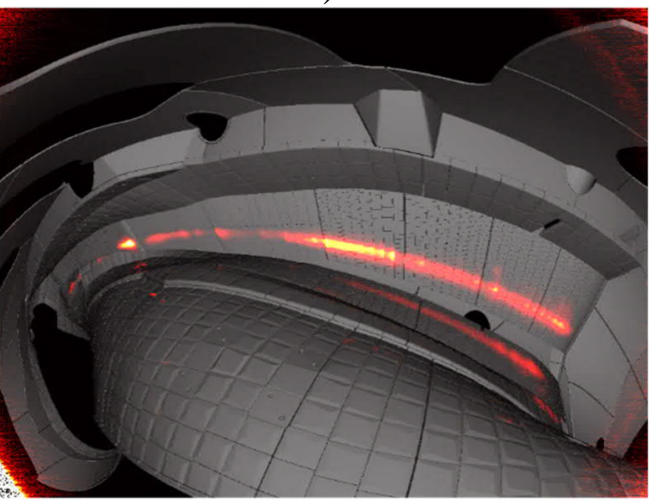

Figure 5 Scene model example. a) CAD view; b) Overlay of the CAD view on the thermal image. 


\section{IV.B CAD-DEPENDENT: THERMAL MAP}

A thermal map is a CAD-based 2D map which unfolds the first wall of the stellarator, including all the PFCs. By means of this map, all the thermal events taken from different cameras can be projected in one single standard grid for monitoring during experiments. The first step for the creation of the thermal map is a transformation from cartesian (Figure 6a) to helical (Figure 6b) coordinates of the stellarator inner surface, by unfolding it with respect to a helical axis and a torsion in the poloidal direction $\vartheta$. Both helical axis and torsion angle change depending on the cross section, i.e. with respect to the toroidal angle $\varphi$.

After this change of coordinates, for each couple of coordinates $\varphi$ and $\vartheta$, two new coordinates $u, v$ are defined, obtaining the thermal map shown in Figure 7a. The coordinates $u$ and $v$ are obtained by line-integrating the surface along the $\varphi$ and $\vartheta$ axes. Figure $7 b$ shows an example of thermal events projected on the thermal map. Given the fact that the torsion angle performs a rotation of $180^{\circ}$ within each module, lower and upper divertors in module 2 and 4 of the thermal map are inverted, i.e. the lower (upper) divertor in module 2 and 4 appears on the top (bottom) of the thermal map.

a)

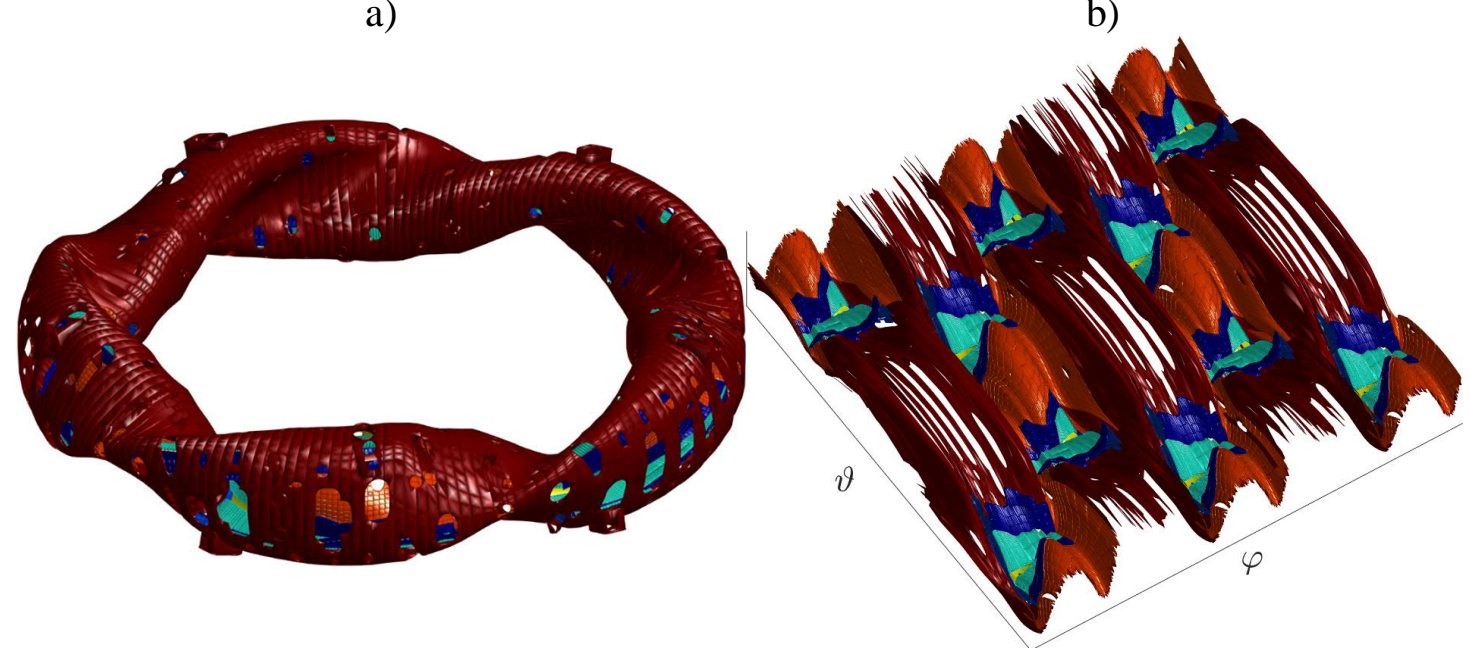

Figure 6 CAD model of the stellarator PFCs in a) cartesian coordinates and b) helical coordinates. 
a)

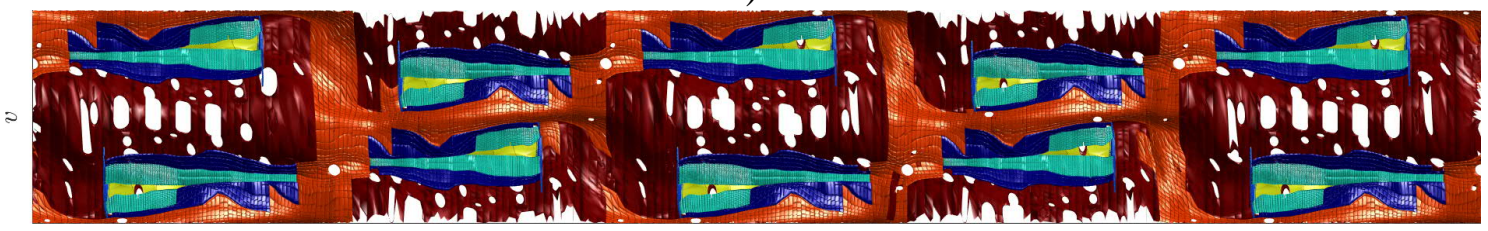

b)

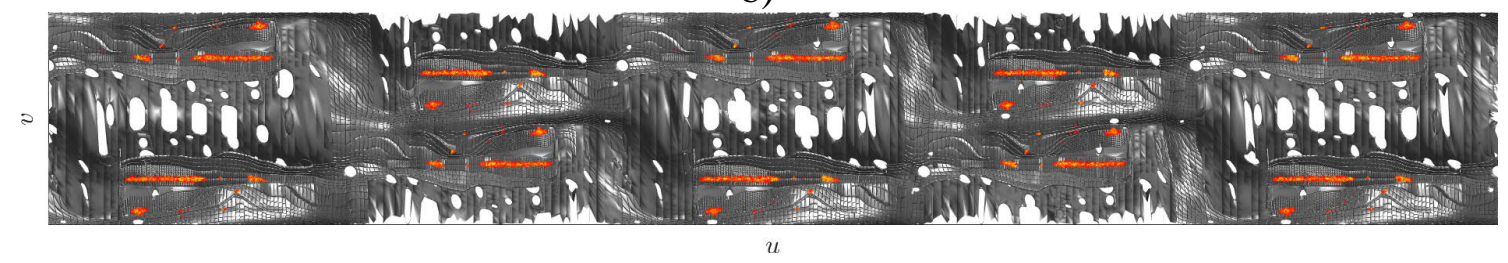

Figure 7 a) Thermal map with different PFCs highlighted with different colors. In order of appearance in the top side (bottom side) from left to right: upper (lower) divertor of module 1, lower (upper) divertor of module 2, upper (lower) divertor of module 3, lower (upper) divertor of module 4, upper (lower) divertor of module 5.

b) Example of thermal events projected on the thermal map.

\section{APPLICATIONS}

These mapping tools are used to relate the detected thermal events on the infrared image with the complex 3D structure of the stellarator PFCs, which is essential to evaluate their risk [4]. The detected thermal events include strike-lines, leading edges, overload hot spots, shine-through hot spots due to the heating systems, fast particle losses and surface layers.

When analysing IR data, thermal events must be identified and characterized by extracting a set of significant properties. These properties or features can then be related with the magnetic configuration and the experimental plasma parameters, and in case of dangerous events, the system can automatically implement the proper control strategies. To this end, it is necessary to implement image processing techniques to segment the IR images, isolating the different thermal events with respect to the background and noise and evaluate their properties. 
Some preliminary results are reported below, regarding the strike-line segmentation and characterization starting from computed heat-flux images.

\section{V.A STRIKE-LINE CHARACTERIZATION}

Figure 8a shows the heat-flux distribution in one of the ten divertors of W7-X. The heat-flux image is constructed by aligning the heat-flux profiles, defined as described in [5], next to each other in a picture. Each vertical line in the heat-flux image corresponds to a single profile. As a first attempt of isolating the strike-line and removing the effect of noise, hot spots and leading edges, a spatio-temporal prefiltering is applied to the image. This prefiltering stage, performed by means of a gaussian spatial filter and of a moving average filter in the time dimension, helps to avoid an over-segmentation of the strike-line. An example of the result is shown in Figure 8b. After this pre-filtering stage, for each profile, i.e. each vertical line in the image, of the divertor target (horizontal or vertical), the strike-line width is estimated, adapting the formula of the wetted area proposed in [12].

In this example, at the end of the process, in standard magnetic configuration [13] it is possible to identify three main objects in the final image (see Figure 8c). These three objects can be analysed and characterized by means of spatial and thermal features and used to give a general characterization of the strike-line in this particular magnetic configuration.

In order to better appreciate the effect of the pre-filtering and of the segmentation procedure, two profiles, one along the y-direction (see Figure 8d) and the other along the x-direction (see Figure 8e), are shown. The position of the two profiles in the heat-flux images is shown as dashed white lines in Figures 8a, 8b and 8c. As it can be noticed, the pre-filtering stage smoothen the profile peak removing the high gradients due to the 
leading edges, while the segmentation stage select from the profile only the relevant information to define the strike-line properties.

a)

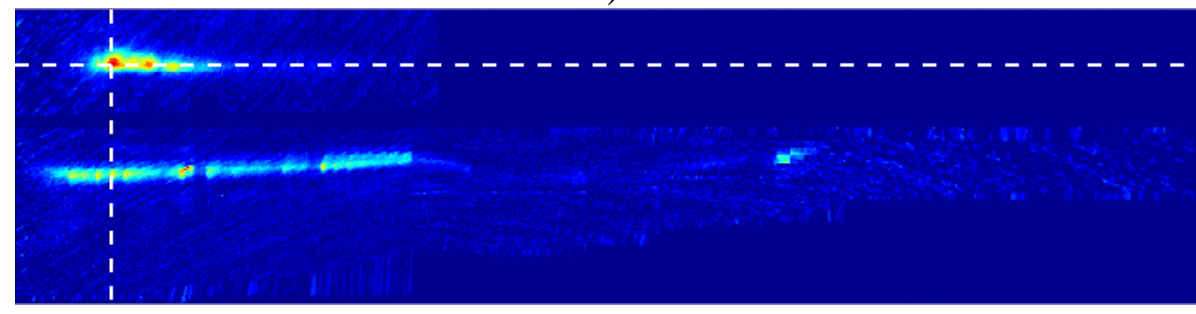

b)

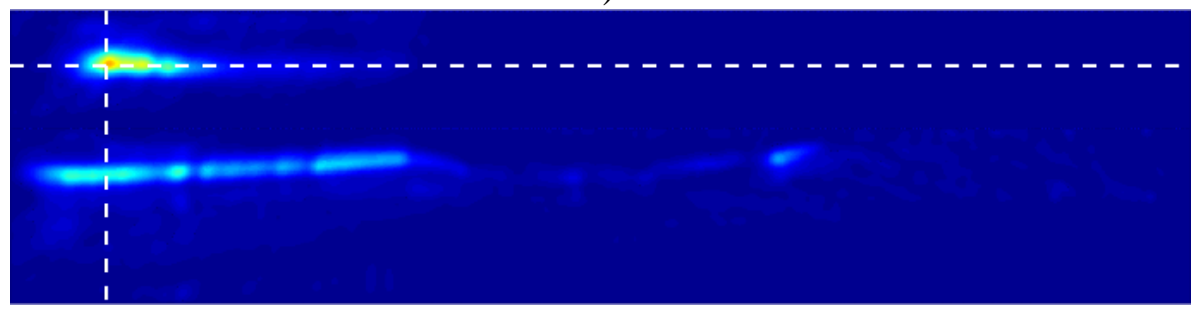

c)
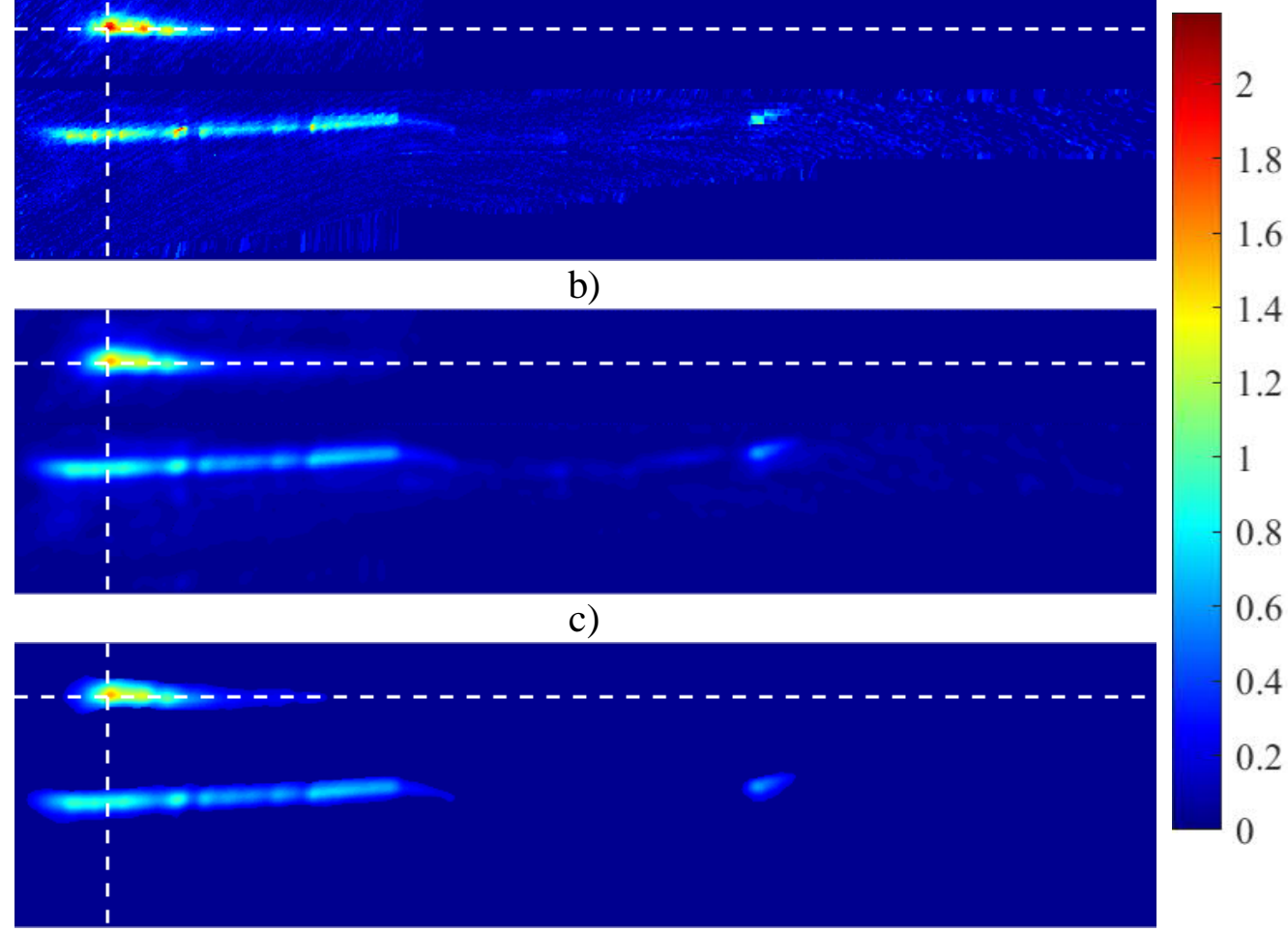

1.6

$-1.4$

d)

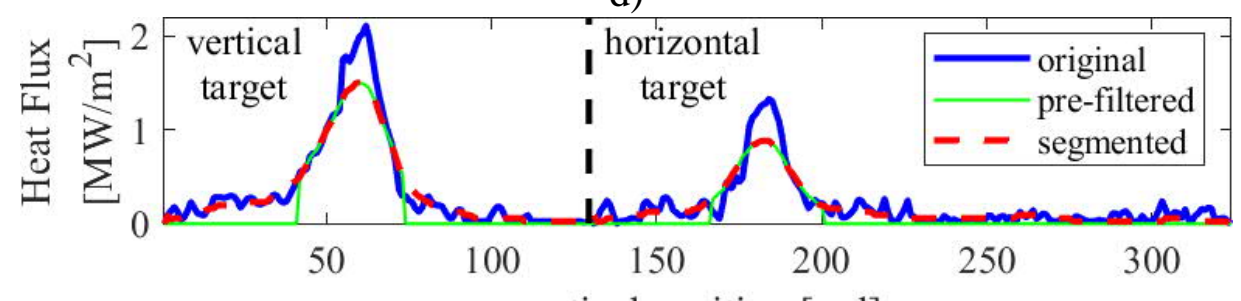

vertical position [pxl]

e)

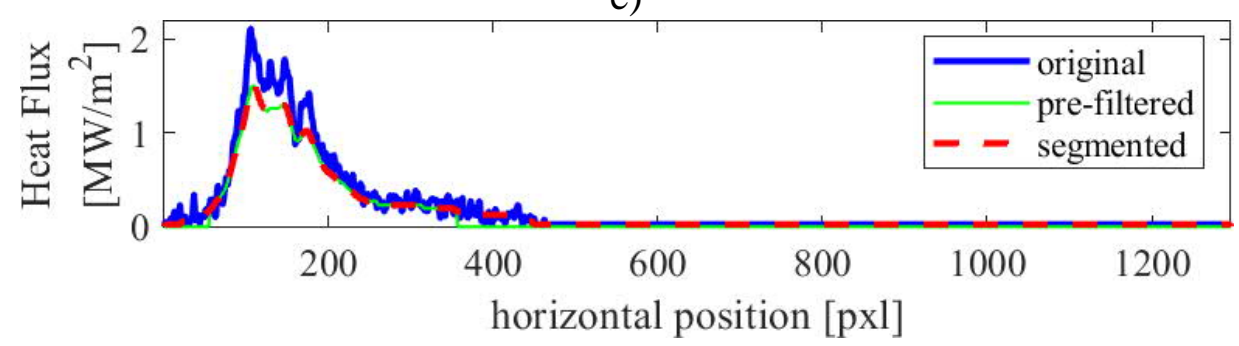

Figure 8. a) Original heat-flux image, b) pre-filtered image, c) final segmented image.

Profiles along d) $y$-direction and e) $x$-direction (see vertical and horizontal dashed white lines in a), b) and c)). 


\section{CONCLUSIONS}

In this paper several tools for the image analysis of the thermographic data are presented. These tools are essentials for the understanding of the infrared images, in particular, for the transformation into surface temperatures and for the calculation of the heat-fluxes, which require a mapping of the data onto the $3 \mathrm{D}$ surfaces of the target components. A lot of work needs to be done in order to automate the procedures in case of camera movements or vibrations, which are possible during next operation of W7-X, but a whole range of possibilities is now open for the analysis of thermographic data. In this context, the characterisation of the heat loads on the PFC targets, as well as the study of their relationship with experimental parameters, together with their monitoring and control for PFC protection, are included.

\section{ACKNOWLEDGMENTS}

This work has been carried out within the framework of the EUROfusion Consortium and has received funding from the Euratom research and training programme 2014-2018 and 2019-2020 under grant agreement No 633053. The views and opinions expressed herein do not necessarily reflect those of the European Commission.

\section{DECLARATION OF INTEREST STATEMENT}

The authors confirm that there are no known conflicts of interest associated with this publication and there has been no significant financial support for this work that could have influenced its outcome. 


\section{REFERENCES}

[1] T. Sunn Pedersen et al., "First divertor physics studies in Wendelstein 7-X", Nuclear Fusion, 59, 9, 096014 (2019).

[2] A. Puig Sitjes, M. Jakubowski, A. Ali, P. Drewelow, V. Moncada, F. Pisano, T. T. Ngo, B. Cannas, J. M. Travere, G. Kocsis, T. Szepesi, T. Szabolics \& W7-X Team, "Wendelstein 7-X Near Real-Time Image Diagnostic System for PlasmaFacing Components Protection", Fusion Science and Technology, 74, 1-2, 116 (2018).

[4] A. Puig Sitjes, Y. Gao, M. Jakubowski, P. Drewelow, H. Niemann, A. Ali, V. Moncada, F. Pisano, T. T. Ngo, B. Cannas, M. Sleczka and W7-X Team, "Observation of thermal events on the plasma facing components of Wendelstein 7-X”, Journal of Instrumentation, 14, C11002 (2019).

[3] M. Jakubowski, P. Drewelow, J. Fellinger, A. Puig Sitjes, G. Wurden, A. Ali, C. Biedermann, B. Cannas, D. Chauvin, M. Gamradt, H. Greve, Y. Gao, D. Hathiramani, R. König, A. Lorenz, V. Moncada, H. Niemann, T. T. Ngo, F. Pisano, T. Sunn Pedersen, and W7-X Team, "Infrared imaging systems for wall protection in the W7-X stellarator", Review of Scientific Instruments, 89, 10, 10E116 (2018).

[5] Y. Gao, M. W. Jakubowski, P. Drewelow, F. Pisano, A. Puig Sitjes, H. Niemann, A. Ali, B. Cannas and W7-X Team, "Methods for quantitative study of divertor heat loads on W7-X”, Nuclear Fusion, 59, 6, 066007 (2019).

[6] D. A. Forsyth, J. Ponce, Computer Vision: a Modern Approach, Prentice Hall PTR (2002).

[7] P. Sturm, "Pinhole Camera Model". In: Ikeuchi K. (eds) Computer Vision, 
Springer, Boston, MA (2014).

[8] F. Pisano, B. Cannas, M. W. Jakubowski, H. Niemann, A. Puig Sitjes, G. A. Wurden and W7-X Team, "Towards a new image processing system at Wendelstein 7-X: From spatial calibration to characterization of thermal events", Review of Scientific Instruments, 89, 12, 123503 (2018).

[9] C. Hughes, M. Glavin, E. Jones, P. Denny, "Review of Geometric Distortion Compensation in Fish-Eye Cameras", IET Irish Signals and Systems Conference (ISSC 2008), Galway, Ireland, June 18-19, 2008, p. 162 (2008).

[10] J. Wang, F. Shi, J. Zhang, Y. Liu, "A new calibration model of camera lens distortion”, Pattern Recognition, 41, 607 (2008).

[11] Z. Zhang, "A flexible new technique for camera calibration," IEEE Trans. Pattern Anal. Mach. Intell., 22, 11, 1330 (2000).

[12] M. W. Jakubowski, A. Ali, P. Drewelow, Y. Gao, K. Hammond, H. Niemann, A. Puig Sitjes, F. Pisano, M. Ślęczka, S. Brezinsek, B. Cannas, M. Endler, R. König, M. Otte, T. S. Pedersen, G. Wurden, D. Zhang and W7-X Team, "3D Heat and Particle Fluxes in Wendelstein 7-X", 27 ${ }^{\text {th }}$ IAEA Fusion Energy Conference (FEC 2018), Gandhinagar, India, October 22-27, 2018 (2018).

[13] T. Andreeva, J. Kisslinger, H. Wobig, "Characteristics of main configurations of Wendelstein 7-X", Problems of Atomic Science and Technology Series: Plasma Physics, 4, 45 (2002). 